\title{
Substâncias que interferem no comportamento de adolescentes: sentidos e significados Socioculturais
}

\author{
Substances that affect adolescents' behavior: socio-cultural meanings
}

\section{Sustancias que interfieren en el comportamiento de adolecentes: sentidos y significados socioambientales}

\author{
Aline Bernard (alinebernard36@yahoo.com.br) \\ Universidade Federal do Rio Grande do Sul- UFRGS \\ Jean Gabriel Regis (jean.regis@sou.unijui.edu.br) \\ Universidade Regional do Noroeste do Estado do Rio Grande do Sul-UNIJUÍ.
}

Eva Teresinha de Oliveira Boff (evaboff@unijui.edu.br)

Universidade Regional do Noroeste do Estado do Rio Grande do Sul-UNIJUí

Resumo: O objetivo desta pesquisa foi compreender as implicações do uso de substâncias alimentares e/ou psicoativas em adolescentes e sua influência na saúde. Foi realizada uma revisão integrativa, pela busca nas bases de dados Capes, PubMed, Lilacs e Medline, no período de 2010 a 2020, com a associação dos descritores: comportamento and adolescentes and hábitos alimentares and ensino; alimentação and adolescentes and substâncias psicoativas; comportamento and adolescentes and cultura and alimentação. Foram encontrados 4.048 artigos. Destes, 262 foram selecionados para leitura na íntegra. Destacamos, neste artigo, uma das categorias que emergiu da análise denominada: "Estilo de vida e hábitos inadequados como facilitadores para comportamentos de risco aos agravos à saúde". A compreensão desta categoria ocorreu a partir das reflexões sobre a relação sociocultural, comportamental e ambiental do referencial teórico de Pierre Bourdieu. Evidenciamos, nas pesquisas, que sedentarismo, hábitos alimentares não saudáveis, uso de substâncias psicoativas e distúrbios alimentares, são comportamentos de risco à saúde. Concluiu-se que grande parte dos estudos que analisam eventos com adolescentes identificou comportamentos de riscos associados com as dimensões socioculturais.

Palavras-chave: Comportamentos; Adolescentes; Substâncias psicoativas

\begin{abstract}
This research aimed to understand the implications of the use of food and/or psychoactive substances in adolescents and their influence on health. An integrative review was carried outthrough search at the databases Capes, PubMed, Lilacs and Medline, from 2010 to 2020, with the association of the descriptors: Behavior and Adolescents and Eating habits and Education; Food and Adolescents and Psychoactive substances; Behavior and Adolescents and Culture and Eating. A total of 4.048 articles were found, of which 262 were selected for full reading. In this article we highlight one of the categories, which emerge from the analysis, called: "Lifestyle and inappropriate habits as facilitators for risky behaviors to
\end{abstract}


health problems". The understanding of this category was based on the reflections about the socio-cultural, behavioral, and environmental relationship of Pierre Bourdieu's theoretical reference. In our researches, it was emphasized that sedentarism, unhealthy eating habits, use of psychoactive substances and eating disorders are risky behaviors to health problems. It was concluded that most studies that analyze events with adolescents identified risk behaviors associated with socio-cultural dimensions.

Keywords: Behavior; Adolescents; Psychoactive substance

Resumen: El objetivo de esta investigación fue comprender las implicaciones del uso dealimentos y/o sustancias psicoactivas em adolescentes y su influencia en la salud. Fue realizada una revisión integradora, mediante la búsqueda en las bases de datos Capes, PubMed, Lilacs y Medline, en el periodo de 2010 a 2020, em asociación a los descriptores: comportamiento AND adolescentes AND hábitos alimentarios AND enseñanza; alimentación AND adolescentes AND sustancias psicoactivas; comportamiento AND adolescentes AND cultura AND alimentación. Fueron hallados 4048 artículos y seleccionados 262 para su lectura completa. Destacamos en este estudio que una de las categorías que surgió del análisis fue: "Estilo de vida y hábitos inadecuados como facilitadores de comportamientos de riesgo para problemas de salud". La comprensión de esta categoría surgió a partir de las reflexiones sobre la relación sociocultural, comportamental y ambiental del referencial teórico de Pierre Bourdieu. En la investigación, comprobamos que la inactividad física, los hábitos alimentarios poco saludables, el uso de sustancias psicoactivas y los trastornos en la alimentación, son conductas de riesgo para la salud. Se concluyo que gran parte de los estudios que analizan eventos con adolescentes identificaron conductas de riego asociadas con las dimensiones socioculturales.

Palabras clave: Comportamientos; Adolescentes; Sustancias psicoactivas

\section{INTRODUÇÃO}

A pesquisa sobre o comportamento de adolescentes e a sua relação com os agravos a saúde, bem como o uso de substâncias alimentares e/ou psicoativas foi realizada a partir de estudos publicados no período de 2010 a 2020 em periódicos científicos, cuja busca ocorreu em julho de 2020. O estudo iniciou-se a partir do interesse dos autores em aprofundar os conhecimentos sobre o consumo de substâncias que interferem na vida escolar de estudantes de educação básica, em especial quanto aos hábitos alimentares e a saúde humana, pois o consumo de substâncias psicoativas, como o álcool, tabaco e drogas ilícitas, inicia, em sua maioria, por adolescentes ainda em idade escolar (SOUSA; PEREIRA, 2018).

A educação alimentar e nutricional também está relacionada com questões ambientais, comportamentais e culturais. O Guia Alimentar para a População Brasileira (BRASIL, 2014), 
apresenta uma ampla discussão, tendo em vista que estão ocorrendo mudanças no padrão alimentar com o aumento do consumo das substâncias industrializadas nos alimentos e diminuição do consumo de alimentos in natura, o que vem aumentando os problemas de saúde, incluindo a obesidade, além de transformar os hábitos alimentares tradicionais e locais (BRASIL, 2014).

Diante disso, a pesquisa aborda uma reflexão sobre a alimentação e às práticas alimentares, contextualizadas no mundo globalizado, com o intuito de construir um diálogo voltado à valorização dos alimentos in natura que fazem parte da história da alimentação e nutrição. Pesquisas de Diez-Garcia e Castro (2011) mostram que a elevada produção e consumo de alimentos ultraprocessados estão levando ao distanciamento da relação das pessoas com os alimentos alterando suas percepções, pensamentos, práticas e ações relacionadas ao ato de comer.

Nesse texto buscamos analisar os estudos com adolescentes identificando comportamentos de riscos associados com as dimensões socioculturais, pois podem implicar em importantes influências no desenvolvimento físico e psicossocial (SANTOS, 2012). A partir da adolescência, o indivíduo passa por um período de experimentação, que pode levar ao aparecimento de comportamentos de risco como: sedentarismo, hábitos alimentares inadequados, distúrbios com a imagem corporal, uso de tabaco, álcool e outras substâncias psicoativas (FARIAS et al., 2009; SANTOS, 2012). Diante das discussões levantadas sobre esse tema, destacamos que tais comportamentos podem levar esses indivíduos a adquirirem problemas de saúde.

Considera-se que a adolescência é uma fase de insegurança nas escolhas, quando ocorrem modificações e a afirmação da identidade, é importante conhecer as implicações do uso abusivo de substâncias psicoativas por adolescentes, e assim auxiliar na sua formação. Com base nos argumentos apontados, o objetivo desta pesquisa foi compreender as implicações do uso de substâncias alimentares e/ou psicoativas em adolescentes e sua influência na saúde.

\section{PRESSUPOSTOS METODOLÓGICOS}

O estado do conhecimento ou da arte foi organizado mediante a busca eletrônica de artigos publicados entre os anos de 2010 e 2020 em periódicos científicos. A busca foi 
realizada em junho de 2020, nas seguintes bases de dados: Capes, Medline, Lilacs, Pubmed. A escolha por esses bancos de dados deu-se por serem espaços de amplo alcance, acesso facilitado e gratuito, e, o mais importante, a qualidade de possuírem periódicos indexados e reconhecidos academicamente.

A busca foi realizada pela identificação dos descritores comportamento and adolescentes and hábitos alimentares and ensino; alimentação and adolescentes and substâncias psicoativas; comportamento and adolescentes and cultura and alimentação (português e inglês). Estabelecemos como critérios de inclusão: estudos que avaliaram a associação entre comportamentos e hábitos alimentares de adolescentes e suas relações com o uso de substâncias psicoativas e aprendizagem escolar, atribuindo significados socioculturais; artigos de livre-acesso e disponíveis na íntegra; artigos completos e revisados por pares; pesquisas realizadas com ambos os sexos; adolescentes de 10 a 19 anos de idade; e artigos publicados nos últimos dez anos (2010 a 2020). Definimos como critérios de exclusão artigos que não se aproximam dos objetivos da pesquisa. $\mathrm{O}$ quadrol expressa o número de trabalhos encontrados de acordo com os descritores elencados para a busca dos dados e suas respectivas bases.

Quadro 1- Relação dos trabalhos encontrados nas plataformas de busca conforme os descritores do estudo

\begin{tabular}{|c|c|c|c|}
\hline \multirow{2}{*}{$\begin{array}{l}\text { Bases de } \\
\text { dados }\end{array}$} & \multicolumn{3}{|c|}{ Números de artigo encontrados com os descritores } \\
\hline & \begin{tabular}{lr}
\multicolumn{3}{c}{ A } \\
Comportamento \\
adolescentes and hábitos \\
alimentares and \\
ensino/Behavior & and \\
adolescent AND & eating \\
habits AND education
\end{tabular} & \begin{tabular}{lr} 
& \multicolumn{1}{c}{ B } \\
Alimentação and adolescentes \\
and & substâncias \\
psicoativas/Food & AND \\
adolescent AND Psychoactive \\
Substances
\end{tabular} & $\begin{array}{l}\text { C } \\
\text { Comportamento and } \\
\text { adolescentes and Cultura and } \\
\text { Alimentação/Behavior AND } \\
\text { adolescent AND culture AND } \\
\text { food }\end{array}$ \\
\hline Capes & 198 & 25 & 227 \\
\hline Medline & 556 & 176 & 560 \\
\hline Lilacs & 71 & 8 & 20 \\
\hline Pubmed & 1.635 & 7 & 565 \\
\hline TOTAL & 2.460 & 216 & 1.372 \\
\hline
\end{tabular}

Fonte: Dados da pesquisa (2020). 
A partir dessa estratégia de investigação encontramos a totalidade de 4.048 estudos. Iniciamos a leitura pelos títulos, e somente 512 artigos tinham aproximação com os objetivos da pesquisa; foi, então, realizada a leitura dos resumos e selecionados 262 trabalhos que continham informações pertinentes aos descritores elencados. Destes apenas 24 artigos foram incluídos ao corpus da análise, os quais estavam diretamente relacionados com a os critérios de inclusão. Destacamos que um artigo presente no corpus de análise é referente a ano pregresso ao determinado na pesquisa e que foi considerado importante à temática, sendo acrescentado com a finalidade de realizar uma reflexão sobre a abordagem utilizada em anos anteriores a respeito do tema estudado nessa revisão do grande número de artigos encontrados. As etapas de seleção dos artigos são ilustradas na Figura 1.

Para análise do corpus utilizamos a Análise Textual Discursiva (ATD) de Moraes e Galiazzi (2016), os quais orientam para um processo de desconstrução, unitarização e categorização. A ATD “corresponde a uma metodologia de análise de informações de natureza qualitativa com a finalidade de produzir novas compreensões sobre os fenômenos e discursos” (p. 13). A ATD "não pretende testar hipóteses para comprová-las ou refutá-las ao final da pesquisa; a intenção é a compreensão, a reconstrução de conhecimentos existentes sobre os temas investigados" (p. 33). Ao definir as categorias e expressá-las "descritivamente a partir dos elementos que as constituem, inicia-se um processo de explicitação de relações entre elas no sentido da construção da estrutura de um metatexto" (MORAES; GALIAZZI, 2016, p. 51).
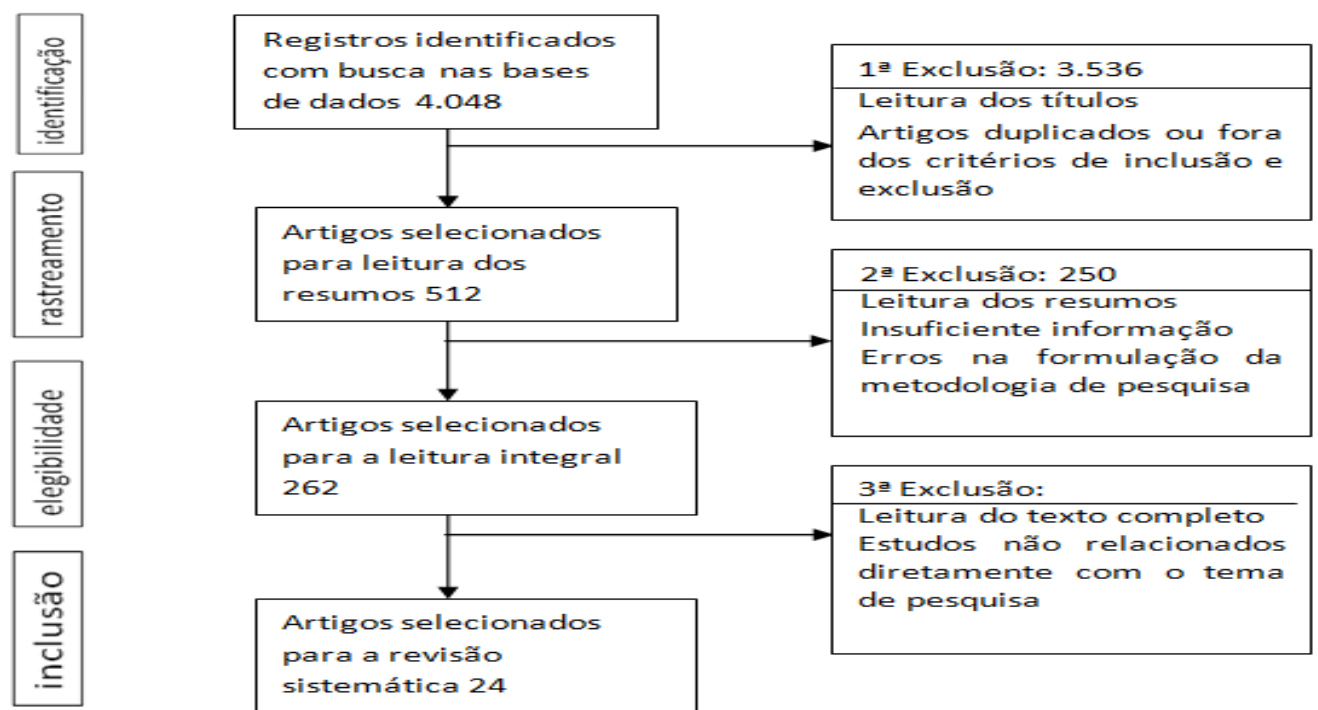

Figura1: Fluxo de identificação, rastreamento, elegibilidade e inclusão dos artigos utilizados 


\section{O processo de descrição e interpretação das produções científicas}

Apresentamos, neste item, os resultados obtidos da análise dos 24 artigos que compuseram o corpus da pesquisa, pois, referente aos descritores da letra A, foram os que tiveram relação direta com o tema de estudo. O Quadro 2 apresenta a síntese com os dados dos artigos selecionados.

Quadro 2-Síntese dos trabalhos encontrados nos periódicos

\begin{tabular}{|c|c|c|c|}
\hline $\mathbf{N}^{\mathbf{O}}$ & Título & Autor & Temática \\
\hline 1 & $\begin{array}{l}\text { Assimetrias regionais. Que diferenças nos estilos } \\
\text { de vida e na satisfação com a vida dos } \\
\text { adolescentes? Um estudo realizado em alunos do } \\
3^{\circ} \text { ciclo do Ensino Básico em Portugal }\end{array}$ & $\begin{array}{l}\text { T. P.F Vieira, } \\
\text { et al (2015) }\end{array}$ & $\begin{array}{l}\text { Diferenças nos estilos de vida e de } \\
\text { satisfação com a vida em estudantes. }\end{array}$ \\
\hline 2 & $\begin{array}{l}\text { Comportamentos de risco à saúde e excesso de } \\
\text { peso corporal em escolares de Toledo, Paraná, } \\
\text { Brasil }\end{array}$ & \begin{tabular}{|l|} 
E. Legnani et \\
al.(2012)
\end{tabular} & $\begin{array}{l}\text { Proporção de adolescentes expostos } \\
\text { aos comportamentos de risco à } \\
\text { saúde. }\end{array}$ \\
\hline 3 & $\begin{array}{l}\text { Comportamento de risco à saúde de adolescentes } \\
\text { escolares }\end{array}$ & \begin{tabular}{|rr} 
F. N. & M. \\
Barbosa & et \\
al. $(2016$ &
\end{tabular} & $\begin{array}{l}\text { Descrever os comportamentos de } \\
\text { risco à saúde de adolescentes. }\end{array}$ \\
\hline 4 & $\begin{array}{l}\text { Fatores associados ao consumo regular de } \\
\text { alimentos obesogênicos: Pesquisa Nacional de } \\
\text { Saúde do Escolar, } 2012\end{array}$ & $\begin{array}{|lr|}\text { G. } & \text { Longo- } \\
\text { Silva } & \text { et } \\
\text { al. }(2016) & \end{array}$ & $\begin{array}{l}\text { Frequência de consumo de alimentos } \\
\text { obesogênicos em adolescentes }\end{array}$ \\
\hline 5 & $\begin{array}{l}\text { Família e proteção ao uso de tabaco, álcool el } \\
\text { drogas em adolescentes, Pesquisa Nacional de } \\
\text { Saúde dos Escolares }\end{array}$ & $\begin{array}{l}\text { D. C. Malta } \\
\text { et al. }(2011)\end{array}$ & $\begin{array}{l}\text { Associação do consumo de } \\
\text { substâncias psicoativas e os fatores } \\
\text { de proteção familiar. }\end{array}$ \\
\hline 6 & $\begin{array}{l}\text { Uso na vida de substâncias ilícitas e fatores } \\
\text { associados entre escolares brasileiros, Pesquisa } \\
\text { Nacional de Saúde do Escolar (PeNSE, 2012) }\end{array}$ & $\begin{array}{l}\text { R. L. Horta et } \\
\text { al. }(2014)\end{array}$ & $\begin{array}{l}\text { Prevalência do uso de drogas ilícitas } \\
\text { entre estudantes e fatores associados }\end{array}$ \\
\hline 7 & $\begin{array}{l}\text { Associação entre comportamento alimentar, } \\
\text { consumo de cigarro, drogas e episódios } \\
\text { depressivos em adolescentes }\end{array}$ & $\begin{array}{l}\text { J. P. Gomes } \\
\text { et al. }(2010)\end{array}$ & $\begin{array}{l}\text { Transtornos alimentares em } \\
\text { estudantes, o hábito de fumar, o uso } \\
\text { de drogas e episódios depressivos. }\end{array}$ \\
\hline 8 & $\begin{array}{l}\text { Autopercepção da imagem corporal e estado } \\
\text { nutricional de escolares }\end{array}$ & $\begin{array}{lr}\text { C. } & \text { R. } \\
\text { Azambuja } & \text { et } \\
\text { al }(2014) & \end{array}$ & Insatisfação com a imagem corporal \\
\hline 9 & $\begin{array}{l}\text { Fatores associados ao comportamento alimentar } \\
\text { inadequado em adolescentes escolares }\end{array}$ & $\begin{array}{l}\text { L. S. Fortes } \\
\text { et al (2013) }\end{array}$ & $\begin{array}{l}\text { Insatisfação corporal, exercicio } \\
\text { fisico e estado nutricional }\end{array}$ \\
\hline & $\begin{array}{l}\text { Uso de álcool, tabaco e outras drogas por } \\
\text { adolescentes escolares em município do Sul do } \\
\text { Brasil }\end{array}$ & $\begin{array}{l}\text { P. C. Vieira } \\
\text { et al.(2008) }\end{array}$ & $\begin{array}{l}\text { Comportamento dos estudantes em } \\
\text { relação a substâncias psicoativas, } \\
\text { estado nutricional e aspectos } \\
\text { emocionais }\end{array}$ \\
\hline 11 & $\begin{array}{l}\text { Comportamentos de risco para transtorno } \\
\text { alimentar: fatores associados em adolescentes } \\
\text { escolares }\end{array}$ & $\begin{array}{l}\text { L. S. Fortes } \\
\text { et al(2013) }\end{array}$ & $\begin{array}{l}\text { Insatisfação corporal, grau de } \\
\text { comprometimento } \quad \text { psicológico, } \\
\text { estado nutricional e nível econômico }\end{array}$ \\
\hline
\end{tabular}




\begin{tabular}{|c|c|c|}
\hline & \begin{tabular}{|l|l|l|}
$\begin{array}{l}\text { Composição e percepção corporal de A. A. Ferreira } \\
\text { adolescentes de escolas públicas }\end{array}$ & & et al(2013) \\
\end{tabular} & $\begin{array}{l}\text { Composição corporal } \\
\text { autopercepção corporal }\end{array}$ \\
\hline 13 & \begin{tabular}{|l|l|}
$\begin{array}{l}\text { Autoestima, imagem corporal e depressão de } \\
\text { adolescentes em diferentes estados nutricionais }\end{array}$ & $\begin{array}{l}\text { A. Rentz- } \\
\text { Fernandes et } \\
\text { al.(2017) }\end{array}$ \\
\end{tabular} & $\begin{array}{l}\text { Autoestima, a imagem corporal e } \\
\text { depressão }\end{array}$ \\
\hline & \begin{tabular}{|l|l|l|} 
Atividade física e hábitos alimentares de J. G. Sousa et \\
adolescentes escolares: pesquisa nacional de \\
saúde do escolar (2019)
\end{tabular} & $\begin{array}{l}\text { Prática de atividade física e o } \\
\text { consumo regular de alimentos } \\
\text { saudáveis e não saudáveis }\end{array}$ \\
\hline & $\begin{array}{l}\text { Relações entre satisfação corporal e psicológica, T. } \\
\text { funcionamento e cognições e comportamentos } \\
\text { relacionados ao peso em adolescentes com } \\
\text { sobrepeso }\end{array}$ & $\begin{array}{l}\text { Satisfação corporal e influências } \\
\text { nos comportamentos }\end{array}$ \\
\hline & \begin{tabular}{|l} 
Representações do corpo: com a palavra um| D. Braga \\
grupo de adolescentes de classes populares
\end{tabular} & Representações sociais do corpo \\
\hline & 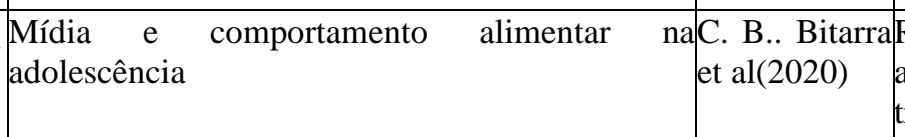 & $\begin{array}{l}\text { Relação entre mídia e escolhas } \\
\text { alimentares na imagem corporal e } \\
\text { transtornos alimentares }\end{array}$ \\
\hline & $\begin{array}{l}\text { Doce veneno: uma análise do consumo de T. R. BalbinoI } \\
\text { bebidas açucaradas por adolescentes }\end{array}$ & $\begin{array}{l}\text { Influência para a redução do } \\
\text { consumo das bebidas açucaradas }\end{array}$ \\
\hline & $\begin{array}{l}\text { Consumo e comportamento alimentar entre R. B. Levy et } \\
\text { adolescentes brasileiros: Pesquisa Nacional de al.(2010) } \\
\text { Saúde do Escolar (PeNSE, 2009) }\end{array}$ & $\begin{array}{lcr}\text { Características } & \text { de consumo } & \text { e } \\
\text { comportamento } & \text { alimentar } & \text { de } \\
\text { adolescentes } & & \end{array}$ \\
\hline 20 & \begin{tabular}{|l|l|} 
Associação transversal entre hábitos alimentares & R. \\
saudáveis e não saudáveis e atividade física de & Fernandes \\
lazer em adolescentes. & al.(2011)
\end{tabular} & $\begin{array}{l}\text { Associação atividade física de lazer } \\
\text { hábitos alimentares }\end{array}$ \\
\hline & $\begin{array}{l}\text { Prevalência e fatores associados ao consumo de L. C. Muniz| } \\
\text { frutas, legumes e verduras entre adolescentes de et al.(2013) } \\
\text { escolas públicas de Caruaru, PE }\end{array}$ & $\begin{array}{l}\text { Prevalência e fatores associados ao } \\
\text { consumo diário de frutas e verduras }\end{array}$ \\
\hline 22 & \begin{tabular}{|l|l|} 
Consumo de açúcares de adição por adolescentes & $\begin{array}{l}\text { M. Braz } \\
\text { em estudo de base populacional }\end{array}$ \\
al.(2019)
\end{tabular} & $\begin{array}{l}\text { Prevalência de ingestão de açúcares } \\
\text { de adição segundo variáveis } \\
\text { demográficas e socioeconômicas. }\end{array}$ \\
\hline 23 & $\begin{array}{l}\text { Comportamento sedentário e consumo de C. S. Costa et } \\
\text { alimentos ultraprocessados entre adolescentes al.(2018) } \\
\text { brasileiros: Pesquisa Nacional de Saúde do } \\
\text { Escolar (PeNSE, 2015) }\end{array}$ & $\begin{array}{|lr|}\text { Comportamento } & \text { sedentário } \\
\text { consumo de } & \text { alimentos } \\
\text { ultraprocessados } & \end{array}$ \\
\hline & $\begin{array}{l}\text { Fatores associados ao consumo de bebidas S. Park } \\
\text { adoçadas com açúcar entre alunos do Ensino al.(2012) } \\
\text { Médio nos Estados Unidos }\end{array}$ & $\begin{array}{l}\text { Características comporta } \\
\text { consumo de bebidas adoça }\end{array}$ \\
\hline
\end{tabular}

\section{Fonte: Os autores (2020).}

O corpus da pesquisa foi constituído por 24 artigos, cuja leitura foi realizada na íntegra sendo agrupados os estudos que abordavam temas semelhantes possibilitando a construção de unidades de significado (consumo de substâncias psicoativas, comportamentos alimentares, alimentos obesogênicos e utraprocessados, meios midiáticos, percepção da imagem corporal, comportamento sedentário). As unidades apresentadas versam sobre o estilo de vida dos adolescentes e ao confrontálas identificamos que todas apresentam comportamentos de risco à saúde. Assim a partir do confronto 
das unidades emergiram as seguintes categorias: estilo de vida e hábitos inadequados como facilitadores para comportamentos de risco aos agravos a saúde. A seguir apresentamos detalhadamente a descrição da categoria de análise emergente do corpus da pesquisa.

\section{RESULTADOS E DISCUSSÕES}

\section{Categoria: Estilo de vida e hábitos inadequados como facilitadores para} comportamentos de risco aos agravos à saúde

A partir da leitura dos artigos que compuseram o corpus desta análise, foi possível verificar que ocorre uma variabilidade entre os estudos e percebe-se a proposição de trabalhos com metodologias diferenciadas nas pesquisas, mas todos os autores mostram que os adolescentes estão expostos a comportamentos de risco e que é importante a sociedade ter conhecimento sobre a questão em foco para poder saber articular melhor as ações envolvidas com os adolescentes. Segundo Vieira et al. (2015), os comportamentos de risco para a saúde, para além da influência direta no fenômeno saúde-doença, no curto prazo têm, também, influência sobre os comportamentos futuros dos indivíduos.

Legnani et al. (2012) identificaram que metade dos adolescentes investigados estavam expostos a comportamentos de risco à saúde; as maiores prevalências foram para prática insuficiente de atividade física, baixo consumo semanal de frutas e vegetais e elevado consumo semanal de refrigerantes e guloseimas, caracterizado como alimentação inadequada.O consumo de substâncias psicoativas também foi identificado, porém em menor proporção. Já um estudo de Vieira et al. (2015) mostrou que os maiores comportamentos de risco para a saúde foram o consumo de bebida alcoólica e outras substâncias psicoativas, alimentação inadequada; a insatisfação com a imagem corporal também foi prevalente. Esses comportamentos de risco, citados por Vieira et al. (2015) e Legnani et al. (2012), foram identificados em praticamente todos os artigos selecionados para esta categoria; portanto, iremos apresentar no decorrer do texto uma análise sobre as circunstâncias desses comportamentos.

Um estudo de Vieira et al. (2008) identificou maior prevalência no consumo ou experimentação de bebida alcoólica, seguida do uso de tabaco e de outras drogas. Neste estudo foram investigados também pais e amigos quanto ao consumo de tabaco e uso de 
álcool. Os resultados revelaram que a maioria dos alunos havia experimentado alguma das substâncias, mencionando que os pais também utilizavam, e uma quantidade significativa de estudantes respondeu que a maioria dos amigos fumava ou bebia, e que a experimentação ocorreu em casa com a família ou com os amigos. Foi evidenciado na pesquisa que o uso de tabaco e álcool pelos pais e pelos amigos está associado com a experimentação dessas substâncias pelos adolescentes (VIEIRA et al., 2008). Logo, percebe-se a relação do meio social em que os jovens estão inseridos para a iniciação de tais comportamentos.

Diante dos dados apresentados, destacamos os sentidos e significados sociais atribuídos a esses comportamentos que afetam diretamente a saúde dos estudantes e esta é um dos temas transversais a ser discutido na escola com interações entre as diferentes áreas do conhecimento, consideramos ser um tema importante para ser dialogado sob a perspectiva das questões socioculturais envolvidas, bem como estar presente em projetos de pesquisa em conjunto com a Universidade.

Continuando a análise sobre a associação entre o consumo de tabaco, álcool, hábitos alimentares e sedentarismo, os autores Barbosa, Casotti e Nery (2016), consideraram os fatores sexo, turno de aula e idade. Nessa análise os autores identificaram que os quatro comportamentos estavam associados ao turno de aula e alguns à idade e ao sexo, mas chamou a atenção de todos os comportamentos estarem associados com o turno de aula, inferindo a influência do grupo nos comportamentos dos adolescentes. Entre os comportamentos de risco, a inatividade física e o baixo consumo de frutas e verduras esteve associado ao turno diurno, enquanto que o maior uso de álcool e tabaco ocorreu nos estudantes que estudavam à noite. Os autores destacaram, ainda, que constataram alta prevalência de comportamentos de risco entre os adolescentes.

Vieira et al. (2015) mencionam que o consumo de álcool está relacionado culturalmente e que surge, muitas vezes, como uma espécie de ritual de transição quase obrigatório, e o primeiro contato dos jovens com o álcool geralmente ocorre no seio da própria família e em casa. Os dados encontrados corroboraram essa visão dos autores, pois os jovens envolvidos em contextos que forneciam recursos positivos eram menos propensos à aquisição de comportamentos de risco e mais propensos ao desenvolvimento de comportamentos protetores da saúde (VIEIRA et al., 2015).Longo-Silva et al. (2016) e Legnani et al.(2012) 
também destacaram que o consumo de cigarros e de bebidas alcoólicas pode estar relacionado à presença de fatores ambientais que são estimulantes ao consumo destas substâncias psicoativas, tais como a existência destes hábitos na família, a influência da mídia, de amigos e o fácil acesso. Longo-Silva et al. (2016) também relacionaram o uso de tabaco e consumo de álcool a práticas de hábitos e estilo de vida pouco saudáveis e que levam a comportamentos alimentares prejudiciais.

Ao refletir sobre as pesquisas discutidas nessa categoria alertamos sobre o consumo de substâncias psicoativas que pode causar problemas psicossociais, emocionais e orgânicos, o tabaco em específico pode provocar sérios problemas respiratórios, doença pulmonar progressiva, acarretando uma diminuição da capacidade vital respiratória, além de prejudicar a saúde do fumante, também, atua de forma coletiva no ambiente em que ele convive (BRASIL, 2017).

Porém, os resultados apresentados sugerem uma tendência ao uso de álcool, tabaco e outras drogas cada vez mais precoce (LEGNANI et al., 2012; VIEIRA et al., 2015; VIEIRA et al., 2008). Realça-se a importância da orientação sobre os efeitos prejudiciais dessas substâncias no início da idade escolar, o que poderia prevenir a experimentação precoce, o uso abusivo na adolescência e em adultos. $\mathrm{O}$ álcool apresentou-se como a substância de maior prevalência, seguido do tabaco (LEGNANI et al., 2012; VIEIRA et al., 2015; VIEIRA et al., 2008). A crença de que a bebida alcoólica não é droga contribui para o estímulo e incentivo ao uso de álcool não somente durante a adolescência, mas em todas as faixas etárias (VIEIRA et al., 2008).

$\mathrm{Na}$ perspectiva desses comportamentos citados pelos autores acima mencionados, apresentamos os estudos de Malta et al. (2011) e Horta et al. (2014) que analisaram a Pesquisa Nacional de Saúde do Escolar (PENSe), ${ }^{1}$ realizada em 2012, e identificaram que, como consequência da busca de independência e da formação da identidade, os amigos passam a exercer um papel cada vez mais importante na vida dos adolescentes. $\mathrm{O}$ excesso de conflitos intrafamiliares contribui para que o adolescente busque nas amizades relações de

${ }^{1}$ PeNSE é uma pesquisa realizada com escolares adolescentes, desde 2009, em parceria como Instituto Brasileiro de Geografia e Estatística (IBGE) e com o apoio do Ministério da Educação do Brasil. 
confiança que não conseguiram estabelecer em casa. Assim, para ser aceito pelo grupo, o jovem pode passar a fazer uso de álcool, tabaco e outras drogas, adquirindo comportamentos e atitudes semelhantes aos do grupo. Com o apoio familiar a necessidade de se identificar com o grupo talvez não seja tão intensa. Por outro lado, a escola é o local onde primeiramente ocorre a formação dos grupos, portanto também exerce papel fundamental no desenvolvimento de comportamentos e hábitos saudáveis nos adolescentes (MALTA et al., 2011; HORTA et al., 2014).

Pensando na importância da escola sobre ações voltadas a saúde de adolescentes evidenciamos a necessidade de formação de professores para dialogar e promover espaços para a reflexão sobre a importância desta temática para a formação dos estudantes. Além disso, compreendemos a formação de professores como um processo contínuo que contribui para o aperfeiçoamento das práticas docentes objetivando a qualidade do ensino.

Seguindo com os comportamentos de risco, encontramos artigos que citam os distúrbios alimentares. O estudo de Gomes et al. (2010) ressalta que o hábito de fumar e consumir drogas apresentou associação significativa com distúrbios alimentares sugestivos de bulimia; distúrbio esse que o autor relaciona com a depressão, encontrando no estudo uma alta prevalência de jovens que relataram episódios depressivos.

Fortes, Morgado e Ferreira (2013), Fortes, Cipriani e Ferreira (2013), Azambuja, Pandolfo e Santos (2014) e Ferreira et al. (2013), associaram a insatisfação corporal com a aquisição de comportamentos alimentares inadequados e prejudiciais à qualidade de vida em adolescentes. Fortes, Morgado e Ferreira (2013) evidenciaram que a insatisfação corporal dos jovens com o peso e a aparência física bem como o comprometimento psicológico, causaram maiores riscos para o desenvolvimento de comportamento alimentar inadequado e, consequentemente, hábitos alimentares não saudáveis. O autor afirma parecer que a gordura corporal acentuada pode ser considerada fator de risco para a instalação dos comportamentos alimentares inadequados no sexo feminino, demonstrando sintomas de compulsão alimentar. Já entre os adolescentes do sexo masculino, com perfil lipídico abaixo do normal, foi identificada restrição da ingestão de alimentos, buscando o aumento da massa muscular. 
Rentz-Fernandes et al. (2016) investigaram a autoestima, a imagem corporal e a depressão, identificando que a insatisfação corporal tem se mostrado mais influente sobre a depressão do que o próprio Índice de Massa Corporal (IMC). Sousa et al. (2019) investigaram os adolescentes e verificaram que o que mais influencia na depressão é o fato de o adolescente se sentir obeso e não realmente ser obeso. Demonstra-se, com isso, a importância de valorizar as subjetividades dos adolescentes.

Ainda dialogando sobre a insatisfação corporal, Cromley et al. (2012) realizaram um estudo identificando que a maior satisfação corporal foi associada a uma menor probabilidade de se envolver em comportamentos não saudáveis de controle de peso, medos menos frequentes de perder o controle sobre e alimentação e menos importância atribuída à magreza. Adolescentes com maior satisfação corporal podem ser protegidos contra os negativos fatores comportamentais e psicológicos associados ao excesso de peso. Com as análises expostas, identificamos que a maioria das evidências disponíveis sugere a insatisfação corporal como um contribuinte-chave para comportamentos alimentares desordenados em adolescentes.

Ao confrontar os artigos analisados percebemos que os estudos não apresentam fatos isolados, pois são identificados que os comportamentos de risco à saúde de adolescentes estão associados entre si. Dessa forma, verificamos o consumo de substâncias psicoativas associados à insatisfação da imagem corporal, o que vem contribuindo com estilos de vida não saudáveis como: controle de peso, excesso de exposição a meios midiáticos, inatividade física, baixo consumo de frutas e vegetais e elevado consumo semanal de refrigerantes e guloseimas.

Os autores Braga, Molina e Figueiredo (2010) e Bittara; Soares (2020) verificaram a relação entre mídia e escolhas alimentares na imagem corporal e no possível desenvolvimento de transtornos alimentares em adolescentes. A mídia tem um papel importante, veiculando ideias de interesse de mercado, caracterizando o mundo ocidental de economia capitalista (BRAGA; MOLINA; FIGUEIREDO, 2010). Os adolescentes são um grupo de risco para o desenvolvimento de transtornos alimentares e a mídia é um fator contribuinte no comportamento alimentar disfuncional (BITTARA; SOARES, 2020). 
Como apresentamos no decorrer da categoria as escolhas alimentares estão relacionadas às percepções de imagem corporal e outros comportamentos de risco, entre eles o comportamento sedentário e hábitos alimentares não saudáveis. Esses temas aparecem na pesquisa de Sousa et al. (2019) e Levy et al. (2010) que analisaram, de forma crítica, os dados da PeNSE realizada em 2009. Sousa et al.(2019) salientam que a exposição dos adolescentes aos meios midiáticos pode promover o consumo de alimentos industrializados enquanto estão assistindo TV ou conectados a outros meios de comunicação, bem como comportamentos sedentários..

Sousa et al. (2019) destacam que a PeNSE demonstrou que os adolescentes entre 13 a 15 anos consomem, ao menos 5 vezes na semana, guloseimas. Sendo um resultado preocupante, pois nessa fase ficou evidente o intenso consumo de alimentos gordurosos, altamente processados e de preparação rápida. Levy et al. (2010) ressaltam que $92 \%$ dos adolescentes haviam consumido guloseimas pelo menos uma vez na semana e que $40 \%$ consumiam refrigerante mais que 5 vezes na semana. Tais hábitos podem culminar em problemas de saúde, como aumento do peso corporal e dificuldade em realizar atividades físicas, predispondo doenças na fase adulta. Com isso, Sousa et al. (2019) concluíram que o consumo de alimentos ultraprocessados ${ }^{2}$, associado à inatividade física, sendo cultivado na infância, repetido na adolescência e, posteriormente, consolidado na fase adulta, leva à projeção do crescimento de Doenças Crônicas Não Transmissíveis (DCNT) para o ano de 2030, necessitando reforçar a reorientação da população sobre o consumo adequado dos alimentos.

O estudo de Fernandes et al. (2011) demonstrou que a prática de esportes culmina na inclusão de alguns de hábitos positivos no estilo de vida do adolescente, incluindo o consumo de alimentos de melhor qualidade. A associação entre comportamentos sedentários e práticas alimentares não saudáveis pode estar relacionada com os dados citados na análise de Sousa $e t$ al. (2019), que relatam o consumo de alimentos industrializados pelos adolescentes enquanto estão conectados aos meios de comunicação, podendo ser explicada pela maior exposição a anúncios publicitários.

\footnotetext{
${ }^{2}$ Formulações industriais à base de ingredientes extraídos ou derivados de alimentos.
} 
Assim como mostra o estudo de Muniz et al. (2013), que relata a relação inversa entre o consumo de alimentos saudáveis e não saudáveis, os adolescentes que consomem alimentos ricos em açúcares e gorduras consomem poucas frutas e vegetais.Um maior consumo de frutas foi observado entre jovens que contaram não ingerir bebidas alcoólicas, o que também foi associado ao maior consumo de arroz e feijão diariamente. Os autores sugerem que comportamentos que caracterizam um estilo de vida saudável seriam praticados de forma conjunta.

Sobre o consumo de bebidas adoçadas, Park et al. (2012), em análise, indicam que quase um em cada quatro estudantes do Ensino Médio relatou consumir bebidas adoçadas pelo menos 3 vezes por dia; além disso, foi associado o consumo com o uso frequente de restaurantes fast-food e tempo prolongado assistindo televisão. Os autores sugeriram no estudo que os adolescentes podem estar consumindo até 3/4 xícaras de açúcar por dia com a ingestão de bebidas adoçadas. Braz et al. (2019) fizeram uma análise sobre os fatores associados ao consumo de açúcares de adição e mostraram uma simultaneidade de comportamentos inadequados, ou seja, os adolescentes que ingerem mais açúcares também apresentam maior tempo de tela, pior qualidade global da dieta e, ainda, identificaram melhor nível socioeconômico associado à maior adição de açúcar.

Balbino e Barbosa (2019) identificaram alguns principais incentivos para o consumo de bebidas açucaradas, entre eles o sabor, o preço, a praticidade e a pressão social dos amigos, quando os adolescentes são impulsionados a consumir para serem aceitos em determinados grupos e situações. Os autores expõem que o sabor das bebidas açucaradas foi um dos principais aspectos relacionados à atratividade do produto.

Como já mencionado a pesquisa mostra que os comportamentos estão diretamente influenciados por fatores socioculturais, socioeconômicos e sociodemográficos. Também indica que as crianças e os adolescentes são influenciados pelos familiares e o meio em que convivem, estando expostos, muitas vezes, a uma alimentação pouco nutricional associada à inatividade física; fatores estes propícios ao sobrepeso e à obesidade.

Além dos comportamentos de risco já apresentado, evidenciamos na pesquisa de Costa et al. (2018) a associação entre dois comportamentos prejudiciais à saúde do adolescente: a 
alta prevalência de consumo diário de, pelo menos, um grupo de alimentos ultraprocessados e de comportamento sedentário maior que 2 horas por dia, posto que em cada dez adolescentes brasileiros sete apresentaram tais comportamentos. Além disso, observou-se associação positiva entre tempo de comportamento sedentário e consumo diário de, ao menos, um grupo de alimentos ultraprocessados, considerando que quanto maior o tempo de comportamento sedentário maior a prevalência de consumo diário de alimentos ultraprocessados. Os autores sugerem estratégias para redução das prevalências dos comportamentos que analisaram, como a regulamentação da oferta de alimentos ultraprocessados em ambiente escolar e a publicidade deste tipo de alimento, com políticas de melhoria do ambiente com a prática de atividades físicas.

Com base nos artigos elencados, podemos perceber que a mídia exerce fator de relevância na escolha dos grupos alimentares, mas não é determinante, uma vez que os grupos sociais nos quais se insere o adolescente também contribuem para sua preferência alimentar. Isso demonstra que os comportamentos dos adolescentes envolvem uma complexidade de fatores relacionados e que é importante que sejam trabalhados em conjunto e não isolados, pois percebemos, também, que os comportamentos não saudáveis se associam e vice-versa.

Diante do exposto, trouxemos para o texto um trabalho realizado por Baumgratz (2019), que também revela o acréscimo do consumo de alimentos industrializados tanto por influência dos grupos em que convivem como pela facilidade e praticidade que os mesmos oferecem e apresentam uma proposta de atividade que pretende impulsionar os alunos a repensar e mudar hábitos adotando uma alimentação e um estilo de vida mais saudável.

Enfim, verificamos nas pesquisas que grande parte dos estudos que analisam eventos com adolescentes mostram a existência de comportamentos de risco associados a sua vida, e estes estão relacionados com as dimensões socioculturais presentes em nossa sociedade. Em razão da alta prevalência de comportamentos de risco entre adolescentes, é fundamental que se realizem estudos sobre o tema com o intuito de conhecer melhor o público adolescente e suas realidades, para, assim, tentar estabelecer políticas adequadas e contribuir para um movimento de promoção da saúde dos adolescentes, visando os benefícios futuros proporcionados pelo desenvolvimento de hábitos saudáveis nessa fase da vida. 
Além disso, consideramos importante o diálogo entre a Educação em Ciências e a Educação em Saúde proporcionando a contextualização e a interdisciplinaridade sobre discussões direcionadas a Educação Alimentar e Nutricional como constitutivas do currículo escolar. Dessa forma, destacamos a abordagem da saúde na escola levando em consideração as dimensões sociais e com aproximação dos estudantes ao proporcionar as discussões voltadas à realidade da sua comunidade.

\section{As contribuições de Bourdieu sobre as representações sociais}

Os estudos incluídos no corpus da análise mostraram que os comportamentos de risco estão relacionados a fatores ambientais, comportamentais e culturais. Dessa forma, trazemos uma reflexão sobre a relação sociocultural, comportamental e ambiental da alimentação, relacionando com os conceitos de Bourdieu, buscando, assim, compreender a categoria discutida na revisão.

A partir da categoria, estilo de vida e hábitos inadequados como facilitadores para comportamentos de risco aos agravos a saúde, identificamos que foi possível analisar as diversidades relacionadas aos comportamentos de risco para a saúde em adolescentes e discuti-las com base em Bourdieu (1983), pois o estilo de vida está relacionado com as disposições e possibilidades encontradas pelo indivíduo em sua classe, e suas escolhas possíveis são proporcionadas por seu habitus, que significa as exterioridades internalizadas pelo indivíduo de acordo com sua trajetória social. Bourdieu (2007) destaca que o habitus é formado durante a socialização do indivíduo, desde o seu relacionamento familiar, sua primeira educação, passando pela escola, religião, trabalho e todos os meios que, enfim, irão contribuir para a sua formação em determinado contexto social. Considera-se que, nas vivências internalizadas, o habitus tende à sua própria conservação, mas pode ser alterado na medida em que se modificam os contatos sociais do indivíduo (BOURDIEU, 1982).

Nesse sentido, Bourdieu (2007) salienta que o conceito de habitus, que é formado na primeira socialização do agente, geralmente na família (considerada uma das esferas de socialização mais importantes), está diretamente vinculado ao pertencimento a uma classe social. Segundo Bourdieu (2007, p.164), “o habitus está relacionado com a capacidade de produzir práticas, com a capacidade de diferenciar e de apreciar essas práticas e os gostos, em que se constitui o mundo social representado, ou seja, o espaço dos estilos de vida”. 
Assim, o habitus alimentar corresponde à adoção de um tipo de prática que tem a ver com costumes estabelecidos tradicionalmente e que atravessam gerações, com as possibilidades reais de aquisição dos alimentos e com uma sociabilidade construída no âmbito familiar e comunitário, sendo compartilhada e atualizada pelas outras dimensões da vida social. Ao alimentar-se de pratos preparados de forma típica, o indivíduo não só dá conta da própria sobrevivência, mas se sente seguro em suas tradições e reconhece sua identidade social.

Nesse sentido, Diez-Garcia e Castro (2011) citam Bourdieu quando ensina que as primeiras referências são da família e são significantes ao constituírem as estruturas das ações dos sujeitos. Dessa forma, cabe considerar a vivência, a realidade, o aprendizado e a experiência proporcionada pelo meio social ao discutir-se os comportamentos, os hábitos alimentares e os estilos de vida dos sujeitos, inclusive dos adolescentes.

Bourdieu (2007) afirma que é o habitus que faz um agente ser detentor de um gosto, porque as preferências estão associadas às condições objetivas de existência. Como a sociedade vem sofrendo fortes influências midiáticas, em conjunto com o aumento da industrialização, os gostos e preferências alimentares estão mudando, oferecendo um contexto que reflete na alimentação e estilo de vida, levando à diminuição do consumo de alimentos in natura, principalmente por jovens, em prol de produtos ultraprocessados, comprometendo não apenas a cultura alimentar, mas, também, podendo afetar a saúde (SILVA; TEIXEIRA; FEREIRA, 2014).

\section{CONSIDERAÇÕES FINAIS}

A partir do processo de análise dos estudos que foram incluídos no corpus da categoria estilo de vida e hábitos inadequados como facilitadores para comportamentos de risco aos agravos à saúde, foi possível identificar alta prevalência destes comportamentos entre adolescentes. Conclui-se que a adolescência é uma fase de novas descobertas e, portanto, torna os adolescentes mais vulneráveis aos comportamentos de risco identificados neste estudo. Os fatores que estão relacionados a tais comportamentos são a aceitação social ou pressão do grupo, a influência familiar, o contexto escolar, as persuasões midiáticas e o contexto social geral em que vivemos atualmente. 
Identificamos, nos trabalhos analisados, que os comportamentos estão relacionados às dimensões sociocultural, subjetiva e individual. Assim, ao discutir-se esse tema em práticas de promoção da saúde, é importante considerar as diversas dimensões que envolvem os comportamentos dos adolescentes, pois estes irão repercutir em suas vidas futuras.

Entendemos que a abordagem no contexto escolar de temas relacionados à saúde dos estudantes vai ao encontro de diretrizes que orientam trabalhar os conteúdos escolares de forma reflexiva e com capacidade de escolhas conscientes, com conteúdos relevantes, que, ao serem discutidos, fazem sentido para os alunos, sendo caracterizados como conteúdos relevantes, aqueles que fazem parte da vida do adolescente, como os comportamentos identificados nesta pesquisa. Dessa forma, acreditamos ser essencial realizar estudos com o intuito de conhecer melhor o público adolescente e suas realidades, para, assim, se tentar estabelecer políticas adequadas e construir um caminho de promoção da saúde deste público, visando os benefícios futuros proporcionados pelo desenvolvimento de hábitos saudáveis nessa fase da vida. 


\section{REFERENCIAS}

AZAMBUJA, C. R.; PANDOLFO,K. C. M.; SANTOS, D. L. Autopercepção da imagem corporal e estado nutricional de escolares. Revista Brasileira de Nutrição Esportiva, São Paulo. v. 8, n. 47, p.316-321,set./out. 2014.

BALBINO, T. R.; BARBOZA, S. I.S. Doce veneno: uma análise do consumo de bebidas açucaradas por adolescentes. Reciis -Rev. Eletron. Comum. Inf. Inov. Saúde, 13(2), p. 365380, abr./jun. 2019.

BARBOSA, F. N. M.; CASOTTI, C. A.; NERY, A. A. Comportamento de risco à saúde de adolescentes escolares. Texto Contexto Enferm., 25(4):e 2620015, 2016.

BAUMGRATZ, C. E.; Czekalski, R. G.; Oliveira, M. S. Hermel, E. E. S. Reflexões sobre o papel da alimentação saudável no contexto escolar. Revista Insignare Scientia. Vol. 2, n. 2. Mai./Ago. 2019.

BITTARA,C.; SOARES, A.Mídia e comportamento alimentar na adolescência. Cadernos Brasileiros de Terapia Ocupacional, 28(1), p. 291-308, 2020.

BOURDIEU, P. A distinção: crítica social do julgamento. São Paulo, SP: Ed. ZOOK, 2007.

BOURDIEU, P. Questões de sociologia. Rio de Janeiro: Ed. Marco Zero, 1983.

BOURDIEU, P.; PASSERON, J.-C. A reprodução: elementos para uma teoria do sistema de ensino. Trad. Reynaldo Bairão. Rio de Janeiro: Francisco Alves, 1982.

BRAGA, P. D.; MOLINA, C. B.; FIGUEIREDO,T. A. M. Representações do corpo: com a palavra um grupo de adolescentes de classes populares. Ciência \& Saúde Coletiva, 15(1), p. 87-95, 2010.

BRASIL. Ministério da Saúde. Proteger e cuidar da Saúde do adolescente na Atenção Básica. Ministério da Saúde: Brasília, 2017.

BRASIL. Ministério da Saúde. Secretaria de Atenção à Saúde. Departamento de Atenção Básica. Guia alimentar para a população brasileira. Brasília: Ministério da Saúde, 2014.

BRAZ, M.; ASSUMPÇÃO, D. B.; AZEVEDO, M. B.; BARROS, F. AZEVEDO,

A. Consumo de açúcares de adição por adolescentes em estudo de base populacional / Added sugar intake by adolescents: A population-based study. Ciênc. Saúde Colet, 24(9): 32373246, set. 2019.

COSTA, C. S.; FLORES, T. R.; GARCIA, A. W. R.; ASSUNÇÃO, M. C.; SANTOS, I. S. Comportamento sedentário e consumo de alimentos ultraprocessados entre adolescentes brasileiros: Pesquisa Nacional de Saúde do Escolar (PeNSE), 2015. Cad. Saúde Pública, 34(3):e00021017, 2018. 
CROMLEY, T. R.;KNATZ, S. R.; ROCKWELL, D.; NEUMARK-SZTAINER, D.; STORY,M.; BOUTELLE, K. Relationships between body satisfaction and psycho logical functioning and weight-related cognitions and behaviors in over weight adolescentes.J. Adolesc. Health, 50(6), p. 651-653, June 2012.

DIEZ-GARCIA, R. W.;CASTRO, I. R. R. A culinária como objeto de estudo e de intervenção no campo da alimentação e nutrição.Ciênc. Saúde Coletiva,[on-line], v.16, n.1, p.91-98, 2011. ISSN 14138123.

FARIAS JÚNIOR, J.C.; NAHAS, M.V.; BARROS, M.V.G.; LOCH, M.R.; OLIVEIRA, E.S.A.; DE BEM, M.F.L.; LOPES, A.S. Comportamentos de risco à saúde em adolescentes no Sul do Brasil: prevalência e fatores associados. Revista Panamericana de Salud Pública,v. 25,n. 4, p. 344-352, 2009.

FERNANDES, R. A.; CHRISTOFARO, D. G. D.; CASONATTO, J.; KAWAGUTI, S. S.; RONQUE, E. R. V.; CARDOSO, J. R.;FREITAS, I. F. J.; OLIVEIRA, A. R. Cross-sectional association between healthy and unhealthy food habits and leisure physical activity in adolescentes. Jornal de Pediatria,v. 87, n. 3, 2011.

FERREIRA, A. A.; NOGUEIRA, J. D.; WIGGERS, I.; FONTANA, K.E.Composição e percepção corporal de adolescentes de escolas públicas.Motricidade,v. 9, n. 3, p. 19-29, 2013.

FORTES, L. S.; MORGADO, F. F. R.; FERREIRA, M. E. C. Fatores associados ao comportamento alimentar inadequado em adolescentes escolares. Rev. Psiq. Clín.,40(2), p. 59-64, 2013.

FORTES,L. S.;CIPRIANI,F. M.; FERREIRA,M. E. C. Comportamentos de risco para transtorno alimentar: fatores associados em adolescentes escolares. Trends Psychiatry Psychother,35(4), p. 279-286, 2013.

GOMES, J. P.; LEGNANI, E.; LEGNANI, R. F. S.; GREGÓRION, P.; SOUZA, R. K. Associação entre comportamento alimentar, consumo de cigarro, drogas e episódios depressivos em adolescentes. Rev. Nutr., Campinas, 23(5), p. 755-762, set./out. 2010.

HORTA, R. L.; HORTA, B. L.;COSTA, A. W. N.da;PRADO, R. R.do;OLIVEIRACAMPOS M.; MALTA, D. C. Uso na vida de substâncias ilícitas e fatores associados entre escolares brasileiros. Pesquisa Nacional de Saúde do Escolar (PeNSE 2012).Rev. Bras. Epidemiol., Suppl.PeNSE, p. 31-45, 2014.

LEGNANI, E. R.F.; LEGNANI, R.A.; DELLAGRANA, M.P.; SILVA, V.C.; BARBOSA FILHO, W. Campos. Comportamentos de risco à saúde e excesso de peso corporal em escolares de Toledo, Paraná, Brasil. Motricidade, v. 8, n. 3, p. 59-70, 2012.

LEVY, R. B. et al.Consumo e comportamento alimentar entre adolescentes brasileiros: Pesquisa Nacional de Saúde do Escolar (PeNSE), 2009. Ciênc. saúde coletiva vol.15 supl.2 Rio de Janeiro Oct. 2010. 
LONGO-SILVA, G.; MENEZES,R. C. E.; SOUZA,C. A. N.; MARINHO,P. M.;BRAZ,M. D. A.; BARROS, M. B.;FILHO, B.Consumo de açúcares de adição por adolescentes em estudo de base populacional. Ciência \& Saúde Coletiva, 24(9), p. 3.237-3.246, 2016.

MALTA, D. C.; PORTO, D. L.; MELO, F. C.; MONTEIRO,R. A.; VASCONCELOS,L. M.; HORTA, S. B.Lessa. Família e proteção ao uso de tabaco, álcool e drogas em adolescentes. Pesquisa Nacional de Saúde dos Escolares. Rev. Bras. Epidemiol.,14(1), Supl.: p. 166-177, 2011.

MORAES, R.; GALIAZZI, M. C. Análise textual discursiva. Ijuí: Editora Unijuí, 2016.

MUNIZ,L. C.; ZANINI, R. V.; SCHNEIDER,B. C.; TASSITANO, R. M.; FEITOSA,W. M. N.; GONZÁLEZ-CHICA, D. A. Prevalência e fatores associados ao consumo de frutas, legumes e verduras entre adolescentes de escolas públicas de Caruaru, PE. Ciência \& Saúde Coletiva, 18(2), p. 393-404, 2013.

PARK S.; BLANCK, H.M.; SHERRY, B.; BRENER, N.; O'TOOLE .T. Factors associeted with sugars weetened beverage intake among United States high school students. J Nutr 2012; 142 (2) :306-312.

RENTZ-FERNANDES, A. R.; SILVEIRA-VIANA, M.; CARLA, M.; ANDRADE,L. Autoestima, imagem corporal e depressão de adolescentes em diferentes estados nutricionais. Revista de Salud Pública,v.19(1), fev.2017.

SANTOS, L. A. S. O fazer educação alimentar e nutricional: algumas contribuições para reflexão. Ciênc. Saúde Coletiva, 17:453-62, 2012.

SILVA, J. G.; TEIXEIRA, M. L. O.; FERREIRA, M. A. Alimentação na adolescência e as relações com a saúde do adolescente. Texto Contexto Enferm., Florianópolis, 23(4), p. 1.095-1.103, out./dez.2014.

SOUSA, D. S. R.; PEREIRA, P. Perfil dos fatores de risco para doenças crônicas nãotransmissíveis em escolares da zona urbana e rural. Revista Brasileira de Prescrição e Fisiologia do Exercício, São Paulo, Vol. 12, №. 72, Jan./Fev. 2018, págs. 38-45.

SOUSA, J. G.; LIMA, L. R.; FERNANDES, C. R. S.; SANTOS, G. M. Atividade física e hábitos alimentares de adolescentes escolares. Pesquisa Nacional de Saúde do Escolar (PeNSE), 2015.Revista Brasileira de Nutrição Esportiva, São Paulo, v. 13, n. 77, p.8793,jan./fev. 2019.

TOLONI, M. H. A.;OLIVEIRA, M. A. A. Fatores associados ao consumo regular de alimentos obesogênicos. Pesquisa Nacional de Saúde do Escolar, 2012.Rev. Nutr., Campinas, 29(5), p. 609-633, set./out. 2016.

TRAVAIN, S. A.; TRAVAIN, C. B. B.; ASSIS, A. As concepções dos alunos do Ensino Fundamental acerca do tema valor calórico dos alimentos e seus impactos na saúde. Revista Insignare Scientia, v.1, n.3, p. 1- 17, 2018. 
VIEIRA, P. C.; AERTS, D. R.; FREDDO, S. L.; BITTENCOURT, A.; MONTEIRO, L.Uso de álcool, tabaco e outras drogas por adolescentes escolares em município do Sul do Brasil. Cad. Saúde Pública, Rio de Janeiro, 24(11), p. 2.487-2.498, nov. 2008.

VIEIRA, T. P. F.; ALVESN, J. C. R.; DIAS, C. S. L.; FONSECA, A. M. L. F. Assimetrias regionais. Que diferenças nos estilos de vida e na satisfação com a vida dos adolescentes? Um estudo realizado em alunos do $3^{\circ}$ ciclo do Ensino Básico em Portugal. Ciência \& Saúde Coletiva, 20(1), p. 17-28, 2015.

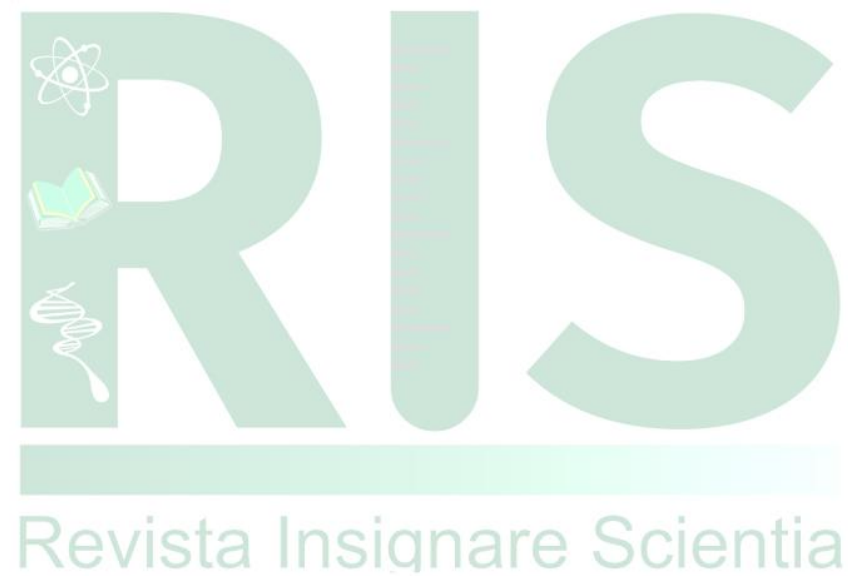

